\title{
Ground State Solutions for a Class of Fractional Differential Equations with Dirichlet Boundary Value Condition
}

\author{
Zhigang Hu, Wenbin Liu, and Jiaying Liu \\ Department of Mathematics, China University of Mining and Technology, Xuzhou 221008, China \\ Correspondence should be addressed to Zhigang Hu; xzhzgya@126.com
}

Received 6 March 2014; Accepted 16 June 2014; Published 1 July 2014

Academic Editor: Micah Osilike

Copyright (C) 2014 Zhigang Hu et al. This is an open access article distributed under the Creative Commons Attribution License, which permits unrestricted use, distribution, and reproduction in any medium, provided the original work is properly cited.

In this paper, we apply the method of the Nehari manifold to study the fractional differential equation $(d / d t)\left((1 / 2){ }_{0} D_{t}^{-\beta}\left(u^{\prime}(t)\right)+\right.$ $\left.(1 / 2){ }_{t} D_{T}^{-\beta}\left(u^{\prime}(t)\right)\right)=f(t, u(t))$, a.e. $t \in[0, T]$, and $u(0)=u(T)=0$, where ${ }_{0} D_{t}^{-\beta},{ }_{t} D_{T}^{-\beta}$ are the left and right Riemann-Liouville fractional integrals of order $0 \leq \beta<1$, respectively. We prove the existence of a ground state solution of the boundary value problem.

\section{Introduction}

Fractional differential equations have played an important role in many fields such as engineering, science, electrical circuits, diffusion, and applied mathematics (see [1-4]). In recent years, some authors have studied the fractional differential equation by using different methods, such as fixed point theorem, coincidence degree theory, and critical point theory (see [5-22]).

By using the Mountain Pass theorem, Jiao and Zhou [17] studied the existence of solutions for the following boundary value problem:

$$
\begin{gathered}
\frac{d}{d t}\left(\frac{1}{2}{ }_{0} D_{t}^{-\beta}\left(u^{\prime}(t)\right)+\frac{1}{2}{ }_{t} D_{T}^{-\beta}\left(u^{\prime}(t)\right)\right) \\
+\nabla F(t, u(t))=0, \quad \text { a.e. } t \in[0, T], \\
u(0)=u(T)=0,
\end{gathered}
$$

where $0<\beta<1,{ }_{0} D_{t}^{-\beta}$ and ${ }_{t} D_{T}^{-\beta}$ are the left and right Riemann-Liouville fractional integrals of order $\beta$, respec- tively, $F:[0, T] \times \mathbb{R}^{N} \rightarrow \mathbb{R}$, and $\nabla F(t, x)$ is the gradient of $F$ with respect to $x$.

By using a critical-points theorem established by G. Bonanno, Bai [19] investigated the following fractional boundary value problem:

$$
\begin{gathered}
\frac{d}{d t}\left(\frac{1}{2}{ }_{0} D_{t}^{\alpha-1}\left({ }_{0}^{C} D_{t}^{\alpha} u(t)\right)-\frac{1}{2}{ }_{t} D_{T}^{\alpha-1}\left({ }_{t}^{C} D_{T}^{\alpha} u(t)\right)\right) \\
+\lambda a(t) f(u(t))=0, \quad \text { a.e. } t \in[0, T], \\
u(0)=u(T)=0,
\end{gathered}
$$

where $\alpha \in(1 / 2,1],{ }_{0} D_{t}^{\alpha-1}$ and ${ }_{t} D_{T}^{\alpha-1}$ are the left and right Riemann-Liouville fractional integrals of order $1-\alpha$, and ${ }_{0}^{C} D_{t}^{\alpha} u(t)$ and ${ }_{t}^{C} D_{T}^{\alpha} u(t)$ are the left and right Caputo fractional derivatives of order $\alpha$.

The authors in [18, 20-22] further studied the existence and multiplicity of solutions for the related problems by critical point theory.

We find that the method of Nehari manifold is seldom used in the above boundary value problem. Inspired by the results in [16-22], we would like to investigate the ground 
state solution for the following fractional boundary value problem:

$$
\begin{array}{r}
\frac{d}{d t}\left(\frac{1}{2}{ }_{0} D_{t}^{-\beta}\left(u^{\prime}(t)\right)+\frac{1}{2}{ }_{t} D_{T}^{-\beta}\left(u^{\prime}(t)\right)\right)=f(t, u(t)), \\
\text { a.e. } t \in[0, T], \\
u(0)=u(T)=0,
\end{array}
$$

where $0<\beta<1,{ }_{0} D_{t}^{-\beta}$ and ${ }_{t} D_{T}^{-\beta}$ are the left and right Riemann-Liouville fractional integrals of order $\beta$, respectively. The technical tool is the method of Nehari manifold. (see $[23,24])$.

This paper is organized as follows. In Section 2, some preliminaries on the fractional calculus are presented. In Section 3, we set up the variational framework of problem (3) and give some necessary lemmas. Finally, Section 4 presents the main result and its proof.

\section{Preliminaries on the Fractional Calculus}

In this section, we will introduce some notations, definitions, and preliminary facts on fractional calculus which are used throughout this paper.

Definition 1 (left and right Riemann-Liouville fractional integrals). Let $f$ be a function defined on $[a, b]$. The left and right Riemann-Liouville fractional integrals of order $\alpha$ for function $f$ denoted by ${ }_{a} D_{t}^{-\alpha} f(t)$ and ${ }_{t} D_{b}^{-\alpha} f(t)$ function, respectively, are defined by

$$
\begin{aligned}
& { }_{a} D_{t}^{-\alpha} f(t)=\frac{1}{\Gamma(\alpha)} \int_{0}^{t}(t-s)^{\alpha-1} f(s) d s, \quad t \in[a, b], \alpha>0, \\
& { }_{t} D_{b}^{-\alpha} f(t)=\frac{1}{\Gamma(\alpha)} \int_{t}^{b}(t-s)^{\alpha-1} f(s) d s, \quad t \in[a, b], \alpha>0,
\end{aligned}
$$

provided that the right-hand side integral is pointwise defined on $[a, b]$.

Definition 2 (left and right Riemann-Liouville fractional derivatives). Let $f$ be a function defined by $[a, b]$. The left and right Riemann-Liouville fractional derivatives of order $\alpha$ for function $f$ denoted by ${ }_{a} D_{t}^{\alpha} f(t)$ and ${ }_{t} D_{b}^{\alpha} f(t)$ function, respectively, are defined by

$$
\begin{aligned}
{ }_{a} D_{t}^{\alpha} f(t) & =\frac{d^{n}}{d t^{n}}{ }_{a} D_{t}^{\alpha-n} f(t) \\
& =\frac{1}{\Gamma(\alpha)} \frac{d^{n}}{d t^{n}} \int_{0}^{t}(t-s)^{n-\alpha-1} f(s) d s, \\
& t \in[a, b], \alpha>0, \\
{ }_{t} D_{b}^{\alpha} f(t) & =(-1)^{n} \frac{d^{n}}{d t^{n}}{ }_{t} D_{b}^{\alpha-n} f(t) \\
& =\frac{(-1)^{n}}{\Gamma(\alpha)} \frac{d^{n}}{d t^{n}} \int_{t}^{b}(s-t)^{n-\alpha-1} f(s) d s, \\
& t \in[a, b], \alpha>0,
\end{aligned}
$$

provided that the right-hand side integral is pointwise defined on $[a, b]$.

Definition 3 (left and right Caputo fractional derivatives). If $\alpha \in(n-1, n)$ and $f \in A C^{n}([a, b], \mathbb{R})$, then the left and right Caputo fractional derivatives of order $\alpha$ for function $f$ denoted by ${ }_{a}^{C} D_{t}^{\alpha} f(t)$ and ${ }_{t}^{C} D_{b}^{\alpha} f(t)$ function, respectively, are defined by

$$
\begin{aligned}
&{ }_{a}^{C} D_{t}^{\alpha} f(t)={ }_{a} D_{t}^{\alpha-n} \frac{d^{n}}{d t^{n}} f(t) \\
&=\frac{1}{\Gamma(\alpha)} \int_{0}^{t}(t-s)^{n-\alpha-1} f^{(n)}(s) d s, \\
& t \in[a, b], \quad \alpha>0, \\
&{ }_{t}^{C} D_{b}^{\alpha} f(t)=(-1)^{n}{ }_{t} D_{b}^{\alpha-n} \frac{d^{n}}{d t^{n}} f(t) \\
&=\frac{(-1)^{n}}{\Gamma(\alpha)} \int_{t}^{b}(s-t)^{n-\alpha-1} f^{(n)}(s) d s, \\
& t \in[a, b], \alpha>0,
\end{aligned}
$$

respectively, where $t \in[a, b]$.

Lemma 4 (see [18]). The left and right Riemann-Liouville fractional integral operators have the property of a semigroup; that is,

$$
\int_{a}^{b}\left[{ }_{a} D_{t}^{-\alpha} f(t)\right] g(t) d t=\int_{a}^{b}\left[{ }_{t} D_{b}^{-\alpha} g(t)\right] f(t) d t, \quad \alpha>0,
$$

provided that $f \in L^{p}([a, b], \mathbb{R}), g \in L^{q}([a, b], \mathbb{R})$ and $p \geq q$, $q \geq 1,1 / p+1 / q \leq 1+\alpha$ or $p \neq 1, q \neq 1,1 / p+1 / q=1+\alpha$.

Lemma 5 (see [18]). Assume that $n-1<\alpha<n$ and $f \in$ $C^{n}[a, b]$. Then,

$$
\begin{gathered}
{ }_{a} D_{t}^{-\alpha}\left({ }_{a}^{C} D_{t}^{\alpha} f(t)\right)=f(t)-\sum_{j=0}^{n-1} \frac{f^{(j)}(a)}{j !}(t-a)^{j}, \\
{ }_{t} D_{b}^{-\alpha}\left({ }_{t}^{C} D_{b}^{\alpha} f(t)\right)=f(t)-\sum_{j=0}^{n-1} \frac{(-1)^{j} f^{(j)}(b)}{j !}(b-t)^{j},
\end{gathered}
$$

for $t \in[a, b]$.

Lemma 6 (see [18]). Assume that $n-1<\alpha<n$. Then,

$$
\begin{array}{r}
{ }_{a}^{C} D_{t}^{\alpha} f(t)={ }_{a} D_{t}^{\alpha} f(t)-\sum_{j=0}^{n-1} \frac{f^{(j)}(a)}{\Gamma(j-\alpha+1)}(t-a)^{j-\alpha,} \\
t \in[a, b], \\
{ }_{t}^{C} D_{b}^{\alpha} f(t)={ }_{t} D_{b}^{\alpha} f(t)-\sum_{j=0}^{n-1} \frac{f^{(j)}(b)}{\Gamma(j-\alpha+1)}(b-t)^{j-\alpha,} \\
t \in[a, b] .
\end{array}
$$




\section{A Variational Setting}

To apply critical point theory for the existence of solutions for boundary value problem (3), we shall state some basic notations and results [18], which will be used in the proof of our main results.

Throughout this paper, we denote $\alpha=1-\beta / 2$ and assume that the following conditions are satisfied.

(H1) $f \in C^{1}(\mathbb{R} \times \mathbb{R})$.

(H2) $f(t, 0)=0=(\partial f / \partial s)(t, 0)$ for every $t \in \mathbb{R}$.

(H3) There are constants $a, b>0$ and $2<p$ such that

$$
\left|\frac{\partial f}{\partial s}(t, s)\right| \leq a+b|s|^{p-2}
$$

for every $t \in \mathbb{R}$ and $s \in \mathbb{R}$.

(H4) There are constants $\mu>2, M>0$ such that

$$
0<\mu F(t, s) \leq s f(t, s)
$$

for all $t \in \mathbb{R}$ and $|s| \geq M$. Here,

$$
F(t, s)=\int_{0}^{s} f(t, x) d x .
$$

(H5) The map $t \rightarrow t^{-1} s f(x, t s)$ is increasing on $(0,+\infty)$, for every $x \in \mathbb{R}$ and $s \in \mathbb{R}$.

Now we construct appropriate function spaces. Denote by $C_{0}^{\infty}([0, T], \mathbb{R})$ the set of all functions $u \in C_{0}^{\infty}([0, T], \mathbb{R})$ with $u(0)=u(T)=0$. The fractional derivative space $E_{0}^{\alpha, p}$ is defined by the closure of $C_{0}^{\infty}([0, T], \mathbb{R})$ with respect to the norm

$$
\|u\|_{\alpha, p}=\left(\int_{0}^{T}|u(t)|^{p} d t+\int_{0}^{T}\left|{ }_{0}^{C} D_{t}^{\alpha} u(t)\right|^{p} d t\right)^{1 / p},
$$

where ${ }_{0}^{C} D_{t}^{\alpha}$ is the $\alpha$-order left Caputo fractional derivative.

Remark 7. If $p=2$, we define $E^{\alpha}=E_{0}^{\alpha, 2}$, with respect to the norm

$$
\|u\|=\left(\int_{0}^{T}|u(t)|^{2} d t+\int_{0}^{T}\left|{ }_{0}^{C} D_{t}^{\alpha} u(t)\right|^{2} d t\right)^{1 / 2} .
$$

The set $E^{\alpha}$ is a reflexive and separable Hilbert space.

Remark 8. For any $u \in E^{\alpha}$, noting the fact $u(0)=0$, we have ${ }_{0} D_{t}^{\alpha} u(t)={ }_{0}^{C} D_{t}^{\alpha} u(t), t \in[0, T]$.

Lemma 9 (see [17]). Let $0<\alpha \leq 1$ and $1<p<\infty$. For all $u \in E_{0}^{\alpha, p}$, one has

$$
\|u\|_{L^{p}} \leq \frac{T^{\alpha}}{\Gamma(\alpha+1)}\left\|_{0}^{C} D_{t}^{\alpha} u\right\|_{L^{p}} .
$$

Moreover, if $\alpha>1 / p$ and $1 / p+1 / q=1$, then

$$
\|u\|_{\infty} \leq \frac{T^{\alpha-1 / p}}{\Gamma(\alpha)[(\alpha-1) q+1]^{1 / q}}\left\|{ }_{0}^{C} D_{t}^{\alpha} u\right\|_{L^{p}} .
$$

According to (15), we can consider $E^{\alpha}$ with respect to the equivalent norm

$$
\|u\|_{\alpha, p}=\left\|{ }_{0}^{C} D_{t}^{\alpha} u\right\|_{L^{p}},\|u\|=\left\|{ }_{0}^{C} D_{t}^{\alpha} u\right\|_{L^{2}} .
$$

Lemma 10 (see [17]). Let $0<\alpha \leq 1$ and $1<p<\infty$. Assume that $\alpha>1 / p$ and the sequence $\left\{u_{k}\right\}$ converges weakly to $u$ in $E_{0}^{\alpha, p}$; that is, $u_{k} \rightarrow u$. Then $u_{k} \rightarrow u$ in $C([0, T], \mathbb{R}) ;$ that is, $\left\|u-u_{k}\right\|_{\infty} \rightarrow 0$ as $k \rightarrow \infty$.

Similar to the proof of [17, Proposition 4.1], we have the following property.

Lemma 11. If $1 / 2<\alpha \leq 1$, for any $u \in E^{\alpha}$, one has

$$
\begin{aligned}
|\cos (\pi \alpha)|\|u\|^{2} & \leq-\int_{0}^{T}\left({ }_{0}^{C} D_{t}^{\alpha} u(t),{ }_{t}^{C} D_{T}^{\alpha} u(t)\right) d t \\
& \leq \frac{1}{|\cos (\pi \alpha)|}\|u\|^{2} .
\end{aligned}
$$

To obtain a weak solution of boundary value problem (3), we assume that $u$ is a sufficiently smooth solution of (3). Multiplying (3) by an arbitrary $v \in C_{0}^{\infty}(0, T)$, we have

$$
\begin{aligned}
-\int_{0}^{T} & \left(\frac{d}{d t}\left(\frac{1}{2}{ }_{0} D_{t}^{-\beta}\left(u^{\prime}(t)\right)+\frac{1}{2}{ }_{t} D_{T}^{-\beta}\left(u^{\prime}(t)\right)\right), v(t)\right) d t \\
& =\int_{0}^{T}(f(t, u(t)), v(t)) d t .
\end{aligned}
$$

Observe that

$$
\begin{gathered}
-\frac{1}{2} \int_{0}^{T}\left(\frac{d}{d t}\left({ }_{0} D_{t}^{-\beta} u^{\prime}(t)+{ }_{t} D_{T}^{-\beta} u^{\prime}(t)\right), v(t)\right) d t \\
=\frac{1}{2} \int_{0}^{T}\left(\left({ }_{0} D_{t}^{-\beta} u^{\prime}(t), v^{\prime}(t)\right)+\left({ }_{t} D_{T}^{-\beta} u^{\prime}(t), v^{\prime}(t)\right)\right) d t \\
=\frac{1}{2} \int_{0}^{T}\left(\left({ }_{0} D_{t}^{-\beta / 2} u^{\prime}(t),{ }_{t} D_{T}^{-\beta / 2} v^{\prime}(t)\right)\right. \\
\left.+\left({ }_{t} D_{T}^{-\beta / 2} u^{\prime}(t),{ }_{0} D_{t}^{-\beta / 2} v^{\prime}(t)\right)\right) d t .
\end{gathered}
$$

As $u(0)=u(T)=v(0)=v(T)=0$, we have

$$
\begin{aligned}
& { }_{0} D_{t}^{-\beta / 2} u^{\prime}(t)={ }_{0} D_{t}^{1-\beta / 2} u(t), \\
& { }_{t} D_{T}^{-\beta / 2} u^{\prime}(t)=-{ }_{t} D_{T}^{1-\beta / 2} u(t), \\
& { }_{0} D_{t}^{-\beta / 2} v^{\prime}(t)={ }_{0} D_{t}^{1-\beta / 2} v(t), \\
& { }_{t} D_{T}^{-\beta / 2} v^{\prime}(t)=-{ }_{t} D_{T}^{1-\beta / 2} v(t) .
\end{aligned}
$$

Then (19) is equivalent to

$$
\begin{aligned}
\int_{0}^{T}- & \frac{1}{2}\left[\left({ }_{0} D_{t}^{\alpha} u(t),{ }_{t} D_{T}^{\alpha} v(t)\right)+\left({ }_{t} D_{T}^{\alpha} u(t),{ }_{0} D_{t}^{\alpha} v(t)\right)\right] d t \\
& =\int_{0}^{T}(f(t, u(t)), v(t)) d t .
\end{aligned}
$$


Since (22) is well defined for $u, v \in E^{\alpha}$, the weak solution of (3) can be defined as follows.

Definition 12. A weak solution of (3) is a function $u \in E^{\alpha}$ such that

$$
\begin{aligned}
\int_{0}^{T}- & \frac{1}{2}\left[\left({ }_{0} D_{t}^{\alpha} u(t),{ }_{t} D_{T}^{\alpha} v(t)\right)+\left({ }_{t} D_{T}^{\alpha} u(t),{ }_{0} D_{t}^{\alpha} v(t)\right)\right] d t \\
& =\int_{0}^{T}(f(t, u(t)), v(t)) d t
\end{aligned}
$$

for every $v \in E^{\alpha}$.

We consider the functional $I: E^{\alpha} \rightarrow \mathbb{R}$, defined by

$$
I(u)=\int_{0}^{T}\left[-\frac{1}{2}\left({ }_{0} D_{t}^{\alpha} u(t),{ }_{t} D_{T}^{\alpha} u(t)\right)-F(t, u(t))\right] d t .
$$

From Theorem 4.1 of [17], we can get that if $1 / 2<\alpha \leq 1$, then the functional $I$ is continuously differentiable on $E^{\alpha}$. Since $I$ is continuously differentiable on $E^{\alpha}$, then

$$
\begin{aligned}
& \left\langle I^{\prime}(u), v\right\rangle \\
& =-\int_{0}^{T} \frac{1}{2}\left[\left({ }_{0} D_{t}^{\alpha} u(t),{ }_{t} D_{T}^{\alpha} v(t)\right)+\left({ }_{t} D_{T}^{\alpha} u(t),{ }_{0} D_{t}^{\alpha} v(t)\right)\right] d t \\
& \quad-\int_{0}^{T}(f(t, u(t)), v(t)) d t
\end{aligned}
$$

for $u, v \in E^{\alpha}$. Hence, a critical point of $I$ is a weak solution of problem (3).

\section{Main Result}

In order to study the solvability of boundary value problem (3), we use the so-called Nehari method. Define

$$
I(u)=-\frac{1}{2} \int_{0}^{T}\left({ }_{0}^{C} D_{t}^{\alpha} u(t),{ }_{t}^{C} D_{T}^{\alpha} u(t)\right) d t-\int_{0}^{T} F(t, u(t)) d t,
$$$$
\forall u \in E^{\alpha},
$$

where $F(t, u)=\int_{0}^{u} f(t, s) d s$.

There is one-to-one correspondence between the critical points of $I$ and weak solutions of boundary value problem (3). Now, we define

$$
\mathscr{N}=\left\{u \in E^{\alpha} \backslash\{0\} \mid\left\langle I^{\prime}(u), u\right\rangle=0\right\} .
$$

Then we know any nonzero critical point of $I$ must be on $\mathcal{N}$. Define

$$
\begin{aligned}
G(u)=\left\langle I^{\prime}(u), u\right\rangle= & -\int_{0}^{T}\left({ }_{0} D_{t}^{\alpha} u(t),{ }_{t} D_{T}^{\alpha} u(t)\right) d t \\
& -\int_{0}^{T}(f(t, u(t)), u(t)) d t .
\end{aligned}
$$

Lemma 13. Assume the hypotheses $\left(H_{1}\right)-\left(H_{5}\right)$ hold. If $u \in \mathcal{N}$ is a critical point of $\left.I\right|_{\mathcal{N}}$, then $I^{\prime}(u)=0$.

Proof. For $u \in \mathcal{N}$, together with $\left(\mathrm{H}_{5}\right)$,

$$
\begin{aligned}
&\left\langle G^{\prime}(u), u\right\rangle=-\int_{0}^{T} 2\left({ }_{0} D_{t}^{\alpha} u(t),{ }_{t} D_{T}^{\alpha} u(t)\right) \\
&+\frac{\partial}{\partial u} f(t, u(t)) \cdot u^{2}(t) \\
& \quad+f(t, u(t)) u(t) d t \\
&= \int_{0}^{T} 2 f(t, u(t)) u(t)-\frac{\partial}{\partial u} f(t, u(t)) \cdot u^{2}(t) \\
&-f(t, u(t)) u(t) d t \\
&= \int_{0}^{T} f(t, u(t)) u(t)-\frac{\partial}{\partial u} f(t, u(t)) \cdot u^{2}(t) d t \\
&<0 .
\end{aligned}
$$

If $u \in \mathcal{N}$ is a critical point of $\left.I\right|_{\mathcal{N}}$, there exists a Lagrange multiplier $\lambda \in \mathbb{R}$, such that $I^{\prime}(u)=\lambda G^{\prime}(u)$. Then we have

$$
\left\langle I^{\prime}(u), u\right\rangle=\lambda\left\langle G^{\prime}(u), u\right\rangle=0 .
$$

By $(29),\left\langle G^{\prime}(u), u\right\rangle \neq 0$, and we have $\lambda=0$. So we can get that $I^{\prime}(u)=0$. The proof is complete.

Lemma 14. Assume the hypotheses $\left(H_{1}\right)-\left(H_{5}\right)$ hold. For any $u \in E^{\alpha} \backslash\{0\}$, there is a unique $y=y(u)$ such that $y(u) u \in \mathcal{N}$ and one has $I(y u)=\max _{y \geq 0} I(y u)>0$.

Proof. First, we claim that there exist constants $\delta>0, \rho>0$ such that $I(u)>0$ for all $u \in B_{\rho}(0) \backslash\{0\}$ and $I(u) \geq \delta$ for all $u \in \partial B_{\rho}(0)$. That is, 0 is a strict local minimizer of $I$. In fact, by $\left(\mathrm{H}_{3}\right)$ we can get that

$$
\forall \varepsilon>0, \exists C_{\varepsilon}>0, \quad|F(t, u)| \leq \frac{\varepsilon}{2}|u|^{2}+C_{\varepsilon}|u|^{p} .
$$

Then together with Lemmas 9 and 11, we have

$$
\begin{aligned}
I(u)= & -\frac{1}{2} \int_{0}^{T}\left({ }_{0}^{C} D_{t}^{\alpha} u(t),{ }_{t}^{C} D_{T}^{\alpha} u(t)\right) d t-\int_{0}^{T} F(t, u(t)) d t \\
\geq & -\frac{1}{2} \int_{0}^{T}\left({ }_{0}^{C} D_{t}^{\alpha} u(t),{ }_{t}^{C} D_{T}^{\alpha} u(t)\right) d t \\
& -\frac{\varepsilon}{2} \int_{0}^{T}|u|^{2} d t-C_{\varepsilon} \int_{0}^{T}|u|^{p} d t \\
\geq & \frac{1}{2}|\cos (\pi \alpha)|\|u\|^{2}-\frac{\varepsilon}{2} \int_{0}^{T}|u|^{2} d t-C_{\varepsilon} \int_{0}^{T}|u|^{p} d t \\
\geq & \left(\frac{1}{2}|\cos (\pi \alpha)|-\frac{\varepsilon}{2} \frac{T^{2 \alpha}}{\Gamma^{2}(\alpha+1)}\right)\|u\|^{2} \\
& -C_{\varepsilon}\left(\frac{T^{p+\alpha-1 / 2}}{\Gamma(\alpha)[(\alpha-1) 2+1]^{1 / 2}}\right)^{p}\|u\|^{p} .
\end{aligned}
$$


Choose $\varepsilon$ such that $(\varepsilon / 2)\left(T^{2 \alpha} / \Gamma^{2}(\alpha+1)\right)=(1 / 4)|\cos (\pi \alpha)|$; then

$$
\begin{aligned}
I(u) \geq & \frac{1}{4}|\cos (\pi \alpha)|\|u\|^{2} \\
& -C_{\varepsilon}\left(\frac{T^{p+\alpha-1 / 2}}{\Gamma(\alpha)[(\alpha-1) 2+1]^{1 / 2}}\right)^{p}\|u\|^{p} \\
= & \|u\|^{2}\left(\frac{1}{4}|\cos (\pi \alpha)|\right. \\
& \left.-C_{\varepsilon}\left(\frac{T^{p+\alpha-1 / 2}}{\Gamma(\alpha)[(\alpha-1) 2+1]^{1 / 2}}\right)^{p}\|u\|^{p-2}\right) .
\end{aligned}
$$

Choose $\rho>0$, such that $C_{\varepsilon}\left(T^{p+\alpha-1 / 2} / \Gamma(\alpha)[(\alpha-1) 2+1]^{1 / 2}\right)^{p}$ $\rho^{p-2}=(1 / 8)|\cos (\pi \alpha)|$. Then we have $I(u) \geq(1 / 8)|\cos (\pi \alpha)|$ $\|u\|^{2}$. Let $\delta=(1 / 8)|\cos (\pi \alpha)|\|u\|^{2}$; then we get that there exist constants $\delta>0, \rho>0$ such that $I(u)>0$ for all $u \in B_{\rho}(0) \backslash\{0\}$ and $I(u) \geq \delta$ for all $u \in \partial B_{\rho}(0)$.

Next, we claim that $I(y u) \rightarrow-\infty$, as $y \rightarrow \infty$. In fact, by $\left(\mathrm{H}_{4}\right)$, there exists a constant $A>0$ such that $F(t, u) \geq A|u|^{\mu}$ for $|u| \geq M$. On the other hand, we can easily get that there exists a constant $B$ such that $F(t, u) \geq B$ for $|u| \leq M$. Then together with Lemma 11, we have

$$
I(y u) \leq \frac{y^{2}}{2|\cos (\pi \alpha)|}\|u\|^{2}-A y^{\mu} \int_{0}^{T}|u|^{\mu} d t-B .
$$

Since $\mu>2$, we can get that $I(y u) \rightarrow-\infty$, as $y \rightarrow \infty$.

Let $g(y):=I(y u)$ for $y>0$. From what we have proved, there has at least one $y_{u}=y(u)>0$ such that

$$
g\left(y_{u}\right)=\max _{y \geq 0} g(y)=\max _{y \geq 0} I(y u)=I\left(y_{u} u\right)
$$

We prove next $g(y)$ has a unique critical point for $y>0$. Consider a critical point

$$
\begin{aligned}
g^{\prime}(y) & =\left\langle I^{\prime}(y u), u\right\rangle \\
& =-\int_{0}^{T} y\left({ }_{0} D_{t}^{\alpha} u,{ }_{t} D_{T}^{\alpha} u\right) d t-\int_{0}^{T} f(t, y u) u d t \\
& =0
\end{aligned}
$$

Then, together with $\left(\mathrm{H}_{5}\right)$, we have

$$
\begin{aligned}
g^{\prime \prime}(y) & =-\int_{0}^{T}\left({ }_{0} D_{t}^{\alpha} u,{ }_{t} D_{T}^{\alpha} u\right) d t-\int_{0}^{T} \frac{\partial f(t, y u)}{\partial(y u)} u^{2} d t \\
& =\int_{0}^{T} \frac{f(t, y u) u}{y} d t-\int_{0}^{T} \frac{\partial f(t, y u)}{\partial(y u)} u^{2} d t \\
& =\frac{1}{y^{2}} \int_{0}^{T} f(t, y u) y u d t-\int_{0}^{T} \frac{\partial f(t, y u)}{\partial(y u)} u^{2} d t \\
& <0 .
\end{aligned}
$$

So we know that if $y$ is a critical point of $g$, then it must be a strict local maximum. This implies the uniqueness.

Finally, from

$$
g^{\prime}(y)=\left\langle I^{\prime}(y u), u\right\rangle=\frac{1}{y}\left\langle I^{\prime}(y u), y u\right\rangle,
$$

we see $y$ is a critical point if $y u \in \mathcal{N}$. Define $m=\inf _{\mathcal{N}} I$. Then we can get that $m \geq \inf _{\partial B_{\rho}(0)} I \geq \delta>0$. The proof is complete.

Lemma 15. Assume the hypotheses $\left(H_{1}\right)-\left(H_{5}\right)$ hold and $m=$ $\inf _{\mathcal{N}} I$. Then there exists $u \in \mathcal{N}$ such that $I(u)=m$.

Proof. We claim that both $I$ and $G$ are weakly lower semicontinuous. In fact, according to Lemma 10 , if $u_{k} \rightarrow u$ in $E^{\alpha}$, then $u_{k} \rightarrow u$ in $C([0, T], \mathbb{R})$. Therefore, $F\left(t, u_{k}(t)\right) \rightarrow F(t, u(t))$ a.e. $t \in[0, T]$. By the Lebesgue dominated convergence theorem, we have $\int_{0}^{T} F\left(t, u_{k}(t)\right) d t \rightarrow \int_{0}^{T} F(t, u(t)) d t$, which means that the functional $u \rightarrow \int_{0}^{T} F(t, u(t)) d t$ is weakly continuous on $E^{\alpha}$. Similarly $u \rightarrow \int_{0}^{T} f(t, u(t)) u(t) d t$ is weakly continuous on $E^{\alpha}$. Since $E^{\alpha}$ is Hilbet space, together with (17) and Lemma 11, we can easily get that $-\int_{0}^{T}\left({ }_{0}^{C} D_{t}^{\alpha} u(t)\right.$, $\left.{ }_{t}^{C} D_{T}^{\alpha} u(t)\right) d t$ is weakly lower semicontinuous on $E^{\alpha}$. Then we get that both $I$ and $G$ are weakly lower semicontinuous.

Since $\mu F(t, u)-u f(t, u)$ is continuous for $t \in[0, T]$ and $|x| \leq M$, there exists $B>0$, such that

$$
F(t, u) \leq \frac{1}{\mu} u f(t, u)+B, \quad t \in[0, T],|x| \leq M
$$

Together with $\left(\mathrm{H}_{4}\right)$, we get

$$
F(t, u) \leq \frac{1}{\mu} u f(t, u)+B, \quad t \in[0, T], x \in \mathbb{R} .
$$

Let $\left\{u_{k}\right\} \in \mathcal{N}$ be a minimizing sequence; that is, $I\left(u_{k}\right) \rightarrow m$, $I^{\prime}\left(u_{k}\right) \rightarrow 0$ as $k \rightarrow \infty$. Then,

$$
\begin{aligned}
m+o(1)= & I\left(u_{k}\right) \\
= & -\frac{1}{2} \int_{0}^{T}\left({ }_{0}^{C} D_{t}^{\alpha} u_{k}(t),{ }_{t}^{C} D_{T}^{\alpha} u_{k}(t)\right) d t \\
& -\int_{0}^{T} F\left(t, u_{k}(t)\right) d t \\
\geq & -\frac{1}{2} \int_{0}^{T}\left({ }_{0}^{C} D_{t}^{\alpha} u_{k}(t),{ }_{t}^{C} D_{T}^{\alpha} u_{k}(t)\right) d t \\
& -\frac{1}{\mu} \int_{0}^{T} u_{k} f\left(t, u_{k}\right) d t-B T \\
= & \left(\frac{1}{\mu}-\frac{1}{2}\right) \int_{0}^{T}\left({ }_{0}^{C} D_{t}^{\alpha} u_{k}(t),{ }_{t}^{C} D_{T}^{\alpha} u_{k}(t)\right) d t
\end{aligned}
$$




$$
\begin{gathered}
+\frac{1}{\mu}\left\langle I^{\prime}\left(u_{k}\right), u_{k}\right\rangle-B T \\
\geq\left(\frac{1}{2}-\frac{1}{\mu}\right)|\cos (\pi \alpha)|\left\|u_{k}\right\|^{2} \\
-\frac{1}{\mu}\left\|I^{\prime}\left(u_{k}\right)\right\|\left\|u_{k}\right\|-B T .
\end{gathered}
$$

By $\mu>2$ and $I^{\prime}\left(u_{k}\right) \rightarrow 0$, we get that $u_{k}$ is bounded in $E$. Since $E^{\alpha}$ is a reflexive space, going to a subsequence if necessary, we may assume that $u_{k} \rightarrow u$ in $E^{\alpha}$. Then from Lemma $10, u_{k} \rightarrow u$ in $C([0, T], \mathbb{R})$. Since $G$ is weakly lower semicontinuous and $\left\{u_{k}\right\} \in \mathcal{N}$, we first have

$$
G(u) \leq \underset{k \rightarrow \infty}{\lim } G\left(u_{k}\right)=0 .
$$

Then we have $u \neq 0$. In fact, if $u=0$, then $u_{k} \rightarrow 0$ in $C([0, T], \mathbb{R})$. By $G\left(u_{k}\right)=0$, we get $\left\|u_{k}\right\| \rightarrow 0$. This is a contradiction with $\left\{u_{k}\right\} \in \mathcal{N}$.

Then from Lemma 14, there exists a unique $y>0$ such that $y u \in \mathscr{N}$. Together with $I$ which is weakly lower semicontinuous, we have

$$
\begin{aligned}
m \leq I(y u) & \leq \varliminf_{k \rightarrow \infty} I\left(y u_{k}\right) \\
& \leq \lim _{k \rightarrow \infty} I\left(y u_{k}\right) \\
& \leq \lim _{k \rightarrow \infty} I\left(u_{k}\right)=m .
\end{aligned}
$$

Then we get that $m$ is achieved at $y u \in \mathcal{N}$. The proof is complete.

Theorem 16. Assuming the hypotheses $\left(H_{1}\right)-\left(H_{5}\right)$ hold, boundary value problem (3) has a weak solution such that $I(u)=m$; that is, boundary value problem (3) has a ground state solution.

Proof. By the Lemmas 14 and 15, we can get that there exists $u \in \mathcal{N}$ such that $I(u)=m=\inf _{\mathcal{N}} I$. Then the $u$ is a critical point of $\left.I\right|_{\mathcal{N}}$. From Lemma 13 we have $I^{\prime}(u)=0$. So boundary value problem (3) has a weak solution such that $I(u)=m$. The proof is complete.

\section{Example}

In this section, we give an example to illustrate our results.

Example 1. Consider the following BVP:

$$
\begin{array}{rlrl}
-\frac{d}{d t}\left(\frac{1}{2}{ }_{0} D_{t}^{-1 / 2}+\frac{1}{2}{ }_{t} D_{T}^{-1 / 2}\right) u^{\prime}(t) & =u^{3}, & t \in[0, T], \\
u(0) & =0, \quad u(T)=0 .
\end{array}
$$

It is easy to verify all the conditions in Theorem 16, so BVP (44) has a ground state solution.

\section{Conflict of Interests}

The authors declare that there is no conflict of interests regarding the publication of this paper.

\section{Acknowledgment}

This research was supported by the Fundamental Research Funds for the Central Universities (2013XK03).

\section{References}

[1] A. A. Kilbas, H. M. Srivastava, and J. J. Trujillo, Theory and Applications of Fractional Differential Equations, vol. 204 of North-Holland Mathematics Studies, Elsevier Science B.V., Amsterdam, The Netherlands, 2006.

[2] K. S. Miller and B. Ross, An Introduction to the Fractional Calculus and Differential Equations, John Wiley \& Sons, New York, NY, USA, 1993.

[3] I. Podlubny, Fractional Differential Equations, Academic Press, San Diego, Calif, USA, 1999.

[4] S. G. Samko, A. A. Kilbas, and O. I. Marichev, Fractional Integral and Derivatives: Theory and Applications, Gordon and Breach, Gordon and Breach, Langhorne, Pa, USA, 1993.

[5] R. P. Agarwal, M. Benchohra, and S. Hamani, "A survey on existence results for boundary value problems of nonlinear fractional differential equations and inclusions," Acta Applicandae Mathematicae, vol. 109, no. 3, pp. 973-1033, 2010.

[6] R. P. Agarwal, D. O’Regan, and S. Staněk, "Positive solutions for Dirichlet problems of singular nonlinear fractional differential equations," Journal of Mathematical Analysis and Applications, vol. 371, no. 1, pp. 57-68, 2010.

[7] B. Ahmad and J. J. Nieto, "Existence results for a coupled system of nonlinear fractional differential equations with threepoint boundary conditions," Computers \& Mathematics with Applications, vol. 58, no. 9, pp. 1838-1843, 2009.

[8] Z. Bai and H. Lu, "Positive solutions for boundary value problem of nonlinear fractional differential equation," Journal of Mathematical Analysis and Applications, vol. 311, no. 2, pp. 495-505, 2005.

[9] Z. Bai and Y. Zhang, "The existence of solutions for a fractional multi-point boundary value problem," Computers \& Mathematics with Applications, vol. 60, no. 8, pp. 2364-2372, 2010.

[10] J. Chen and X. H. Tang, "Existence and multiplicity of solutions for some fractional boundary value problem via critical point theory," Abstract and Applied Analysis, vol. 2012, Article ID 648635, 21 pages, 2012.

[11] B. Ge, "Multiple solutions for a class of fractional boundary value problems," Abstract and Applied Analysis, vol. 2012, Article ID 468980, 16 pages, 2012.

[12] W. Jiang, "The existence of solutions to boundary value problems of fractional differential equations at resonance," Nonlinear Analysis: Theory, Methods \& Applications, vol. 74, no. 5, pp. 1987-1994, 2011.

[13] S. H. Liang and J. H. Zhang, "Positive solutions for boundary value problems of nonlinear fractional differential equation," Nonlinear Analysis: Theory, Methods and Applications, vol. 71, no. 11, pp. 5545-5550, 2009.

[14] S. Zhang, "Existence of solution for a boundary value problem of fractional order," Acta Mathematica Scientia B, vol. 26, no. 2, pp. 220-228, 2006. 
[15] S. Zhang, "Existence of a solution for the fractional differential equation with nonlinear boundary conditions," Computers \& Mathematics with Applications, vol. 61, no. 4, pp. 1202-1208, 2011.

[16] V. J. Ervin and J. P. Roop, "Variational formulation for the stationary fractional advection dispersion equation," Numerical Methods for Partial Differential Equations, vol. 22, no. 3, pp. 558576, 2006.

[17] F. Jiao and Y. Zhou, "Existence of solutions for a class of fractional boundary value problems via critical point theory," Computers \& Mathematics with Applications, vol. 62, no. 3, pp. 1181-1199, 2011.

[18] F. Jiao and Y. Zhou, "Existence results for fractional boundary value problem via critical point theory," International Journal of Bifurcation and Chaos, vol. 22, no. 4, Article ID 1250086, 17 pages, 2012.

[19] C. Bai, "Existence of three solutions for a nonlinear fractional boundary value problem via a critical points theorem," Abstract and Applied Analysis, vol. 2012, Article ID 963105, 13 pages, 2012.

[20] C. Bai, "Existence of solutions for a nonlinear fractional boundary value problem via a local minimum theorem," Electronic Journal of Differential Equations, vol. 2012, no. 176, pp. 1-9, 2012.

[21] Y. Li, H. Sun, and Q. Zhang, "Existence of solutions to fractional boundary-value problems with a parameter," Electronic Journal of Differential Equations, vol. 2013, no. 141, 12 pages, 2013.

[22] H. R. Sun and Q. G. Zhang, "Existence of solutions for a fractional boundary value problem via the Mountain Pass method and an iterative technique," Computers \& Mathematics with Applications, vol. 64, no. 10, pp. 3436-3443, 2012.

[23] Z. Nehari, "On a class of nonlinear second-order differential equations," Transactions of the American Mathematical Society, vol. 95, pp. 101-123, 1960.

[24] Z. Nehari, "Characteristic values associated with a class of nonlinear second-order differential equations," Acta Mathematica, vol. 105, pp. 141-175, 1961. 


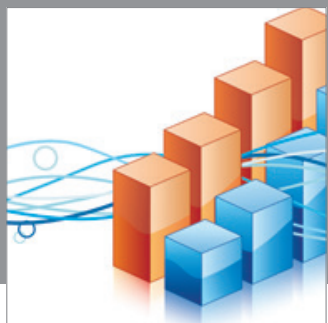

Advances in

Operations Research

mansans

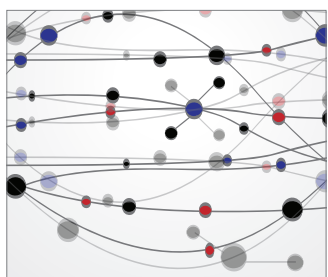

The Scientific World Journal
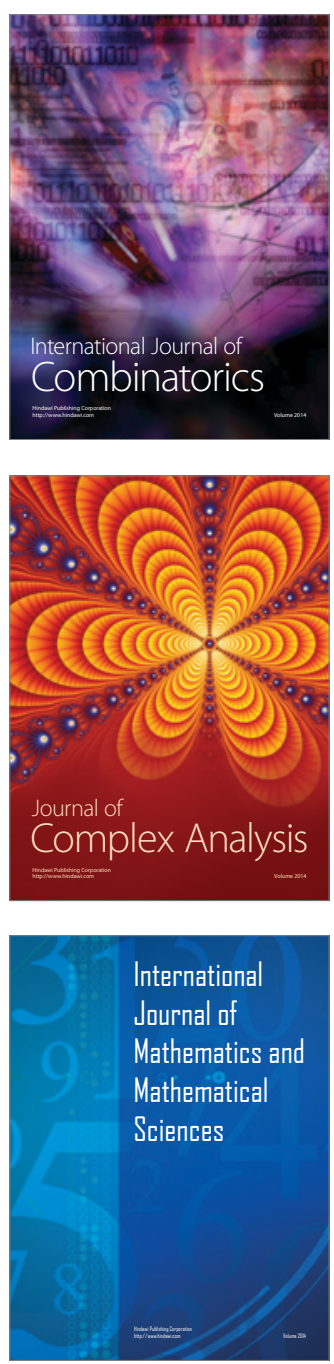
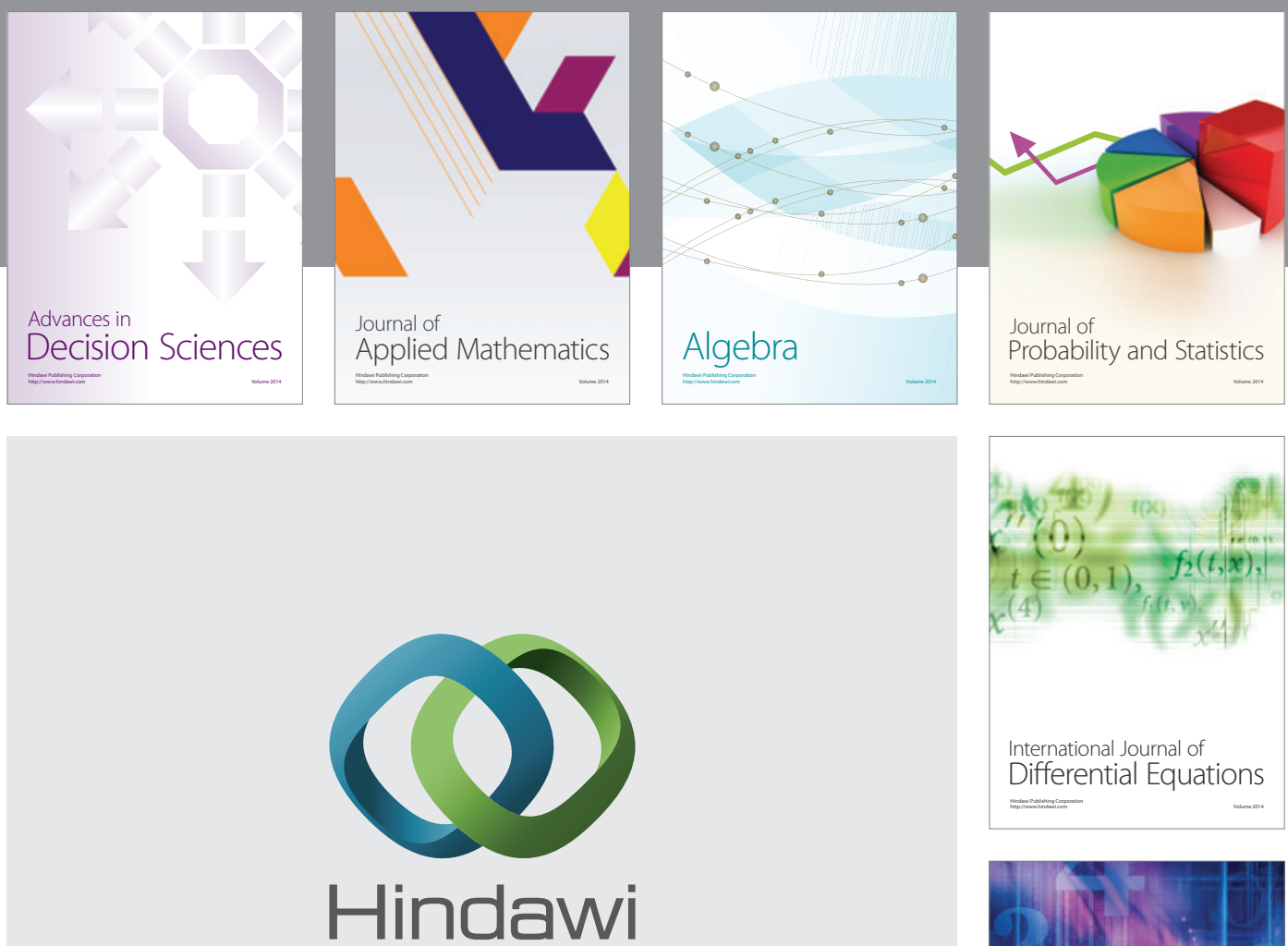

Submit your manuscripts at http://www.hindawi.com
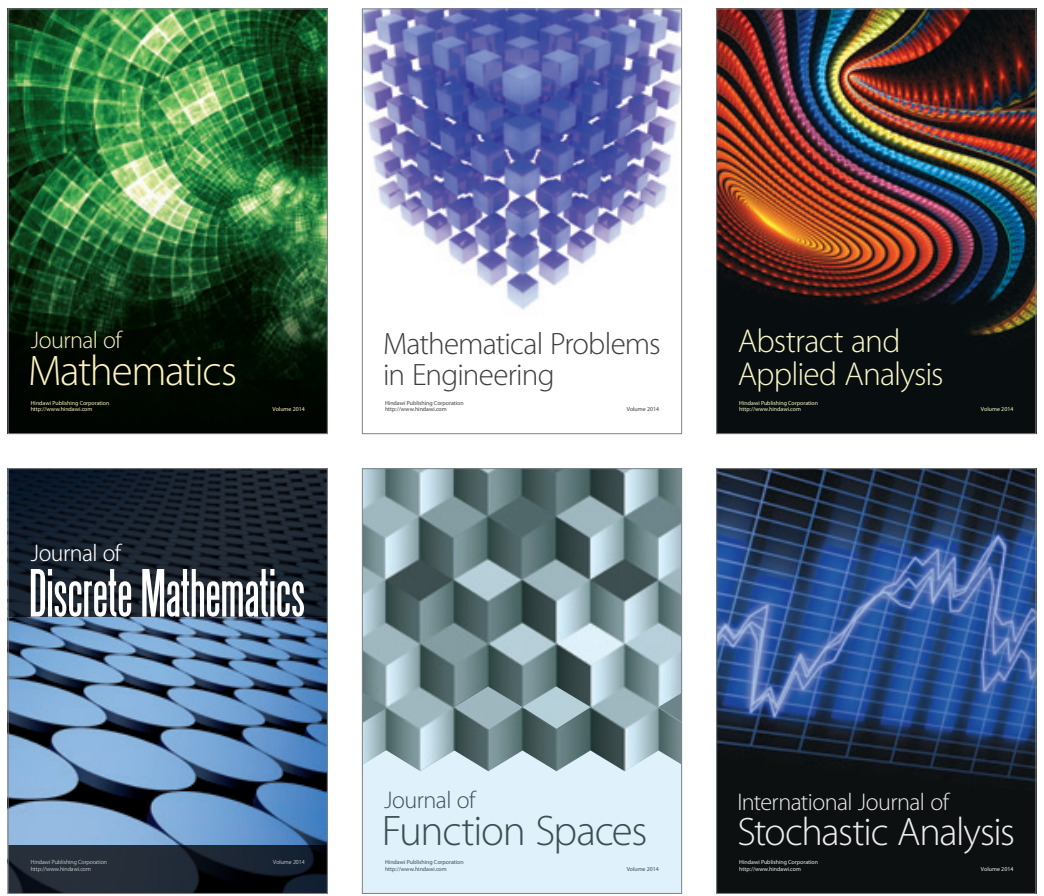

Journal of

Function Spaces

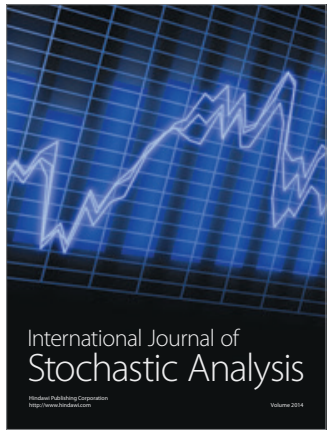

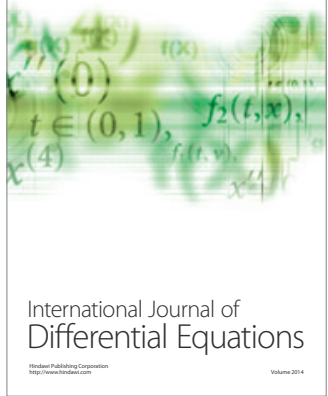
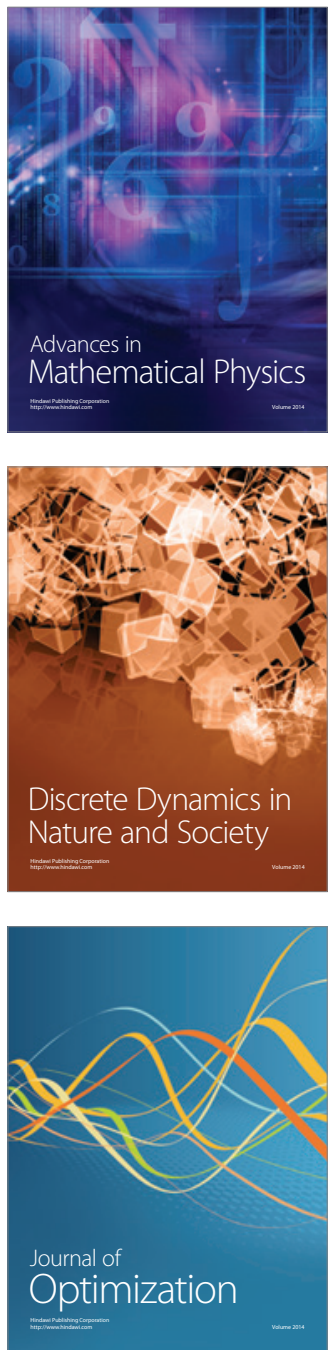